\section{What is IUGS?}

The International Union of Geological Sciences is one of the largest and most active scientific non-governmental organisations in the world. Founded in 1961, IUGS is a member of the International Council of Scientific Unions (ICSU). IUGS promotes and encourages the study of geological problems, especially those of world-wide significance, and supports and facilitates international. multidisciplinary cooperation and dialogue in the earth sciences.

The IUGS Commissions. Committees and Boards are concerned with a wide range of geological rescarch of direct interest to governments. industry and academic institu lions within the earth sciences. Major effort is directed towards international Joint Programmes sponsored by IUGS and other organisations. Affiliated organisations are large autonomous international associations which share with IUGS an interest in planning and undertaking activities and meetings of mutual benefit.

Membership of IUGS is open to countries generally through a designated adhering organisation' which may be a national committee or some other national earth science body or institution. Each adhering organisation pays an annual subscription according to its category of membership. A special class of membership (under four categories) has been established to enable industry and national and international bodies (both governmental and nongovernmental) as well as individuals, to belong to IUGS and to participate, to various degrees, in the planning of IUGS activities according to their calegory of membership.

The ultimate authority for the management of JUGS is vested in the Council which is composed of representatives from the national adhering organisations. It usually meets every four years during the International Geological Congress to review progress by IUGS constituent bodies, the administration of the Union and its accounts, and to decide on any new proposals or initiatives.

Overall management is the responsibility of the Executive Committee which is elected by Council on the recommendation of a Nominating Committee. The Executive Committee normally meets annually and deals with major policy, operational and administrative issues affecting the Union. Current members of the Executive Committee are listed on the inside front cover together with the adhering members. Further information about IUGS may be obtained from :

\section{The IUGS Secretariat}

$\%$ Geological Survey of Norway

PO Box 3006 Lade

$\mathrm{N}-7002$ Trondheim

Norway

\section{COMMISSIONS}

COMMISSION ON COMPARATIVE PLANETOLOGY (CCP)

Dr A T Basilevsky, Chairman

Vernadsky Institute, Kosygmst. 19, 117975

GSP-1, Moscow. RUSSIA

COMMISSION ON THE MANAGEMENT AND APPLICATION OF GEO. SCIENCE INFORMATION (COGEOINFO)

Dr James E Biesecker, Chairman

US Geological Survey, 801 National Center.

Reston, VA 22092, USA

COMMISSION ON GEOSCIENCE FOR ENVIRONMENTAL PLANNING (COGEOENVIRONMENT)

Dr F Chr Wolff, Chairman Geological Survey of Norway, PO Box 3006 Lade. N-7002 Trondheim, NORWAY

COMMISSION FOR GEOSCIENCE EDUCATION AND TRAINING (COGEOED) Dr P Cooray, Chairman

426 Mahakanda Road. Hindagala, Kandy District. SRI LANKA

\section{COMMISSION ON GI.OBAL, SEDIMEN-}

TARY GEOLOGY

Dr Robert N Ginsburg, Chairman

University of Miami. RSMAS/MGG, 460)

Rickenbacker Causeway, Miami.

FI. 33149-1098, USA

\section{COMMISSION ON THE HISTORY OF} GEOLOGICAL SCIENCES (INHIGEO) Professor David F Branagan. Chairman Department of Geology and Geophysics. University of Sydney, NSW 2006, AUSTRALIA

\section{COMMISSION ON IGNEOUS AND} METAMORPHIC PETROGENESIS (CIMP)

Dr Ian Camphell, Chairman

The Australian National University, Institute of Advanced Studies, GPO Box 4, Canberra, ACT 2601. AUSTRALIA

COMMISSION FOR MARINE GEOLOGY (CMG)

Professor Dr Y Lancelot, Chairman

Laboratoire de Géologie du Quaternaire, CNRS Luminy, Case 907, F-13288 Marseille Cedex 9. FRANCE

COMMISSION ON STRATIGRAPHY (ICS)

Professor J Remane, Chairman

Université de Neuchâtel Institut de Géologie, 11 rue Emile-Argand. $\mathrm{CH}-2000$ Neuchâtel 7. SWITZ.ERI AND

\section{COMMISSION ON SYSTEMATICS IN} PETROLOGY

Professor Jörg Keller, Chairman Mineralogisch-petrographisches Institut der Universität, Alberstralie 23b, D-79104 Freiburg, GERMANY

\section{COMMISSION ON TECTONICS} (COMTEC)

Dr P Hancock, Chairman

Department of Geology, University of Bristol, Wills Memorial Building, Queens Road, Bris tol BS\& IRJ, UK

\section{COMMISSION ON FOSSIL FUELS (CFF)}

Dr R Sinding-Larsen, Chairman Norwegian Institute of Technology, Department of Geology, Høgskoleringen 6, N-7034 Trondheim, NORWAY
ADVISORY BOARDS

ADVISORY BOARD FOR PUBLICATIONS $(\mathrm{ABP})$

Dr W G E Caldwell

Department of Geology, University of Western Ontario, London. Ontario N6A 5B8, CANADA

ADVISORY BOARD FOR RESEARCH DEVELOPMENT (ABRD)

Vacancy

AFFILIATED ORGANIZA. TIONS

ASSOCIATION OF ARAB GEOLOGISTS

(AGA)

Dr Wissam S Al-Hashami, Secretary General, PO Box 1247, Baghdad 12 I 2, IRAQ

ASSOCIATION OF EXPLORATION GEOCHEMISTS (AEG)

Dr Sherman P Marsh. Secretary

US Geological Survey. Federal Center. MS 973. Denver, CO 80225, USA

THE AMERICAN ASSOCIATION OF PETROLEUM GEOLOGISTS

Dr Gary D Howell, Science Director and International Development Advisor

The American Association of Petroleum Geologists, PO Box 979, 1444 South Boulder. Tulsa, OK 74101-0979, USA

ASSOCIATION OF EUROPEAN GEOLOGICAL SOCIETIES (AEGS)

Dr B Deffontaines, Secretary

Société Géologique de France T26.16-1E Boite 129. Dept. Geotectonics-LGGST, 4 Place Jussicu, F-75252 Paris Cedex 05 FRANCE

ASSOCIATION OF GEOSCIENTISTS FOR INTERNATIONAL DEVEIOPMENT (AGID)

AGID Headquarters, Institute of Geosciences, University of São Paulo, CP 20.899, 01498-970 São Paulo, BRAZIL

ASSOCIATION INTERNATIONALE POUR L'ÉTUDE DES ARGILES (AIPEA) Professor R. Schoonheydt, Secretary General COK Katholieke Universiteit Leuven, Kardinaal Mercierlaan 92, B-3001 Heverlee, BELGIUM

ASSOCIATION DES SERVICES GEOIOGIQUES AFRICAINS (ASGA)

Dr Jean-Claude Bidet, Technical Secretary $\%$ CIFEG, BRGM, Avenue de Concyr, BP 6517, 45065 Orléans Cedex 2, HRANCE

CARPATHIAN BALKAN GEOLOGICAL ASSOCIATION (CBGA)

Dr Kristina Kolceva, Secretary

Faculty of Geology and Geography, Sofia University, 1504 Sofia, BULGARIA

CIRCUM-PACIFIC COUNCIL FOR ENERGY AND MINERAI RESOURCES Dr Michel T Halbouty, President and Chairman The Halbouty Center, 5100 Westheimer Road, Houston, TX 77056, USA

COMMISSION FOR THE GEOLOGICAL MAP OF THE WORLD (CGMW) Dr Phillipe Bouysse, Secretary General Maison de la Géologie, 77, rue Claude Bernard, F-75005 Paris, FRANCE

EUROPEAN ASSOCIATION OF SCIENCE FDITORS (FASE)

Ms Maeve O'Connor, Secretary Treasurer 
49 Rossendale Way, London NW 1 0XB. UK GEOCHEMICAL SOCIETY (GS)

Dr Steven B Shirey, Secretary

The Geochemical Society, Department of Terrestrial Magnetism, Carnegie Institution of Washington, 5241 Broad Branch Road NW, Washington, DC 20015, USA

\section{GEOLOGICAL SOCIETY OF AFRICA} (GSAF)

Mrs A Amoako-Mensah, General Secretary Industrial Research Instilute, PO Box M32 Accra, GHANA

\section{GEOLOGICAL SOCIETY OF AMERICA} (GSAm)

Dr Donald Davidsen, Executive Director Geological Society of America. PO Box 9140, Boulder. CO 80301. USA

INTERNATIONAL ASSOCIATION OF ENGINEERING GEOLOGY (IAEG) Dr L. Primel. Secretary General

Laboratoire Central des Ponts et Chaussess.

58 Boulevard Lefehvre. F-757.32 Paris Cedex 15, FRANCF

INTERNATIONAL, ASSOCIATION OF GEOCHEMISTRY AND COSMOCHEMISTRY (IAGC)

Dr Mel Gascogne, Secretary

AECL Research, Whiteshell Laboralories.

Pinawa, Manitoba ROE II.0, CANADA

INTERNATIONAL ASSOCIATION ON THE GENESIS OF ORE DEPOSITS (LAGOD) Ing. Jaroslav Aichler, Secretary General Czech Geological Survey, PO Box 6579001 Jesenik, CZECH REPUBLIC

INTERNATIONAL ASSOCIATION OF GEOMORPHOLOGISTS

Dr Robert J Allison, Secretary General Department of Geography. University of Durham, South Road, Durham DHI 3I.E. UK

INTERNATIONAL ASSOCIATION OF HYDROGEOLOGISTS (IAH)

Dr Andrew Skinner, Secretary Gencral PO Box 9, Kenilworth, Warwickshire CV8 IJG. UK

INTERNATIONAL ASSOCIATION FOR MATHEMATICAL GEOLOGY (IAMG) Dr Michael Ed Hohn. Secretary West Virginia Geological Survey, PO Box 879 Morgantown, WV 26505, USA

INTERNATIONAL ASSOCIATION OF SED. IMENTOLOGISTS (IAS)

Dr F Surlyk. Sccretary General

Geologisk Centralinstitut, $\varnothing_{\text {ster Voldgade } 10,}$ DK-1350 Copenhagen, DENMARK

INTERNATIONAL ASSOCIATION OF STRUCTURAL/TECTONIC GEOLOGISTS Dr Sue H Treagus, Convenor

Department of Geology, University of Manchester, Manchester M13 9PL, UK

INTERNATIONAL, CENTER FOR TRAINING AND EXCHANGES IN GEO. SCIENCES (CIFEG)

Dr Jean-Claude Napias, Director

International Center for Training and Exchanges in the Geosciences, Avenue de Concyr, BP 6517, 45065 Orléans Cedex 2, FRANCE

INTERNATIONAL FEDERATION OF PALYNOLOGICAL SOCIETIES (IFPS)
Dr Owen K Davis, Secretary Treasurer Department of Geoscience, University of Arizona, Tucson, Arizona 85721. USA

INTERNATIONAL MINERAIOGICAL ASSOCIATION (IMA)

Dr S S Hafner. Secretary

Institute of Mineralogy, University of Marburg. Hans-Meerwein-Straß3e, D-35032 Marbura GERMANY

INTERNATIONAL PALAEONTOLOGICAI ASSOCIATION (IPA)

Professor D L. Bruton. Secretary General

Universitet i Oslo, Sarsgate 1. N-0562 Oslo, NORWAY

INTERNATIONAL PERMAFROST ASSOCIATION

Dr Jerry Brown, Secretary General

PO Box 9200. Arlington, VA 22219-0200. USA

INTERNATIONAL, SOCIETY FOR ROCK

MECHANICS

Dr Jose Delgado Rodrigues, Secretary General I aboratório Nacional de Engenharia Civil 10l 10) Avenida do Brasil, P-1799 Lisboa Codex. PORTUGAL

INTERNATIONAI, SOCIETY FOR SOIL MECHANICS ANID FOUNIATTION ENGINEERING

Dr R H G Parry, Secretary General

I niversily Lingineering Department, Trumpington Strect. Cambridge CB2 1P\%, UK

INTERNATIONAL UNION FOR QUATERNARY RESEARCH (INQUA)

Dr lidward Derbyshire, Secretary Department of Geography, Royal Holloway. University of London, Egham, Surrey TW20 ()EX, UK

THE METEORITICAL SOCIETY

Dr Monica M Grady. Secretary

Natural History Museum, Cromwell Road,

London SW7 5BD. UK

\section{SOCIETY OF ECONOMIC GEOLOGISTS}

(SFCi)

John A Thoms, Executive Secretary

5808 South Rapp Street. Suite 209, Little Creck

One Building, Littleton, CO 80120-1942. USA

SOCIETY FOR GEOLOGY APPIAED TO

MINERAI, DEPOSITS (SC;A)

Dr Maurice Pagel, Execulive Secretary

CREGU BP-23, F-54501 Vandauve les

Nancy, FRANCE

SOCIETY FOR SEDIMENTARY GEOLOGY (SEPM)

Ms Robin Dixon, Executive Director PO Box 4756. Tulsa, OK 74159, USA

JOINT PROGRAMMES

30TH INTERNATIONAI, GEOI OGICAI, CONGRESS (1996)

Professor Zhang Hongren, Secretary General. 30th IGC. PO Box 823, Beijing 100037 CHINA

GEOLOGICAL APPLICATION OF REMOTE SENSING (GARS) Dr C C Weber, Chairman BRGM. BP' 6009, F-45060 Orléans Cedex 02, FRANCE
CIRCUM-ATIANTIC PROJECT (CAP)

Dr John A Reinemund, Coordinator

PO Box 890, Leesburg, VA 22075, USA

INTERNATIONAI, COUNCII, OF SCIENTIFIC UNIONS (ICSU)

Dr J Marton-I efevre, Executive Secretary ICSU Secretariat, 51 Boulevard de Mont morency, F-75601 Paris, FRANCE.

INTERNATIONAI, GEOLOGICAI, CORREI.ATION PROGRAMME (IGCP) Dr Vladislav Babuška, Secretary, UNESCO. Division of Earth Sciences, 1 ruc Miollis, F-757.32 Paris, FRANCE

INTER-UNION COMMISSION ON THE I. ITHOSPHERE (ICL)

Dr Kevin C Burke, President University of Houston. Deparment of Geosciences, Houston, TX 77204-5503, USA

IUGS/UNESCO PROJECT ON MINERAI DEPOSIT MODELLING

Dr Gabor Gaál, Chairman DMP, Hungarian Geological Survey, Stetania ut 14, PO Box 106, H-1442 Budapest, HUNGARY

\section{ASSOCIATE MEMBERS}

AUSTRALIAN COUNCII, OF CHAIRMEN OF EARTH SCIENCES

Atın: Prof. Ian R Plimer. President, University of Newcastle Department of Geology, Rankin Drive Newcastle. NSW, 2308 AUSTRAI IA

\section{AUSTRALIAN GEOIOGICAI, SURVEY} ORGANISATION

GPO Box 378. Canberra, ACT 2601. AUSTRALIA

\section{EI-ROGRINI}

Universidade Federal do Para, Cx. Postal 1611, 66000 Belcin-PA. BRAZIL

GEOLOGICAL SOCIETY OF SOUTH AFRICA

Attn: Mrs L Burger. Secretary. PO Box 44283 , 2104 Linden, SOUTH AFRICA

KING ABIUULAZIZ CITY FOR SCIENCF AND TECHNOIOCY

Attn: Dr Abderrahman A-Sayed. Directorate of Technology Transfer, PO Box 6086, Riyadh 11442, SAUDI ARABIA

DR G A MORI

Viale Ugo Ojetti 23, 50137 Firen/e, ITALY

NORTHERN TERRITORY GEOI OGICAI SURVEY

Attn: Dr Patul Le Messurier, Director, Darwin. NT OSO1. AUSTRALIA

OII. \& NATURAL GAS COMMISSION (ADMIPE)

Attn: Dr Jagadish Pandey, Geology Deparment, Geoscience Research Division, 9 Kadulagarth Road, Dehra Dun - 248195. INDIA

SIOOVAK BUREAU OF GEOLOGY

Atn: Dr Oswald Trtilek.

Vice Director of Secretariat.

POBax 6?

81005 Bratislava 15.

Bukarestska,

Slovak Republic 